\title{
Simple Method for Evaluating Singular Integrals
}

\author{
Nhan T. Tran \\ Department of Mathematics, Kansas State University, Manhattan, USA \\ Email:nhantran@ksu.edu
}

How to cite this paper: Tran, N.T (2017) Simple Method for Evaluating Singular Integrals. American Journal of Computational Mathematics, 7, 444-450. https://doi.org/10.4236/ajcm.2017.74032

Received: November 12, 2017

Accepted: December 11, 2017

Published: December 14, 2017

Copyright (C) 2017 by author and Scientific Research Publishing Inc. This work is licensed under the Creative Commons Attribution International License (CC BY 4.0).

http://creativecommons.org/licenses/by/4.0/

\begin{abstract}
In this paper, we develop a method for evaluating one dimensional singular integrals (weakly, strongly, and hyper-singular) that converge in the sense of Cauchy principal value and Hadamard finite part integrals. A proof of convergence of this method is also provided.
\end{abstract}

\section{Keywords}

Singular Integral, Weakly Singular, Strongly Singular, Hyper Singular, Numerical Integration

\section{Introduction}

Many problems in engineering and science require evaluating singular integrals. The problem considered in this paper is of practical interest in many areas. For example, in electromagnetic and acoustic wave scattering, the boundary integral equations have singular kernels, see [1]-[6]. In fluid and solid mechanics, physicists and engineers face the same problem, see [7] [8]. Thus, the study of such integrals plays an important role in engineering and science.

The one dimensional singular integrals are defined in the literature as follows

$$
\int_{a}^{b} \frac{u(t)}{(t-s)^{p}} \mathrm{~d} t, \quad s \in(a, b), \quad p>0,
$$

in which $u(t)$ is a continuous function. These integrals are classified by the order of singularity. If $p<1$, the integral is called weakly singular. If $p=1$, the integral is strongly singular. If $p>1$, the integral is called hyper-singular, see [9]. In other words, an integral is called weakly singular if its value exists and continuous at the singularity. An integral is called strongly singular if both the integrand and integral are singular. An integral is called hyper-singular if the 
kernel has a higher-order singularity than the dimension of the integral. For strongly singular integrals, they are often defined in terms of Cauchy principal value, see [10]. For hyper singular integrals, they are often interpreted as Hadamard finite part integrals, see [11].

There are many special methods developed to treat singular integral problems since numerical integration routines often lead to inaccurate solutions. For example, to deal with the singularities in surface integral equations, the method of moments regularizes the singular integrals by sourcing them analytically for specific observation point [12] [13]. Other methods include Gaussian quadrature method which has high-order of accuracy with a non-uniform mesh [14] [15], Newton-Cotes method which has low-order of accuracy with a uniform mesh [16] [17] [18], Guiggiani's method which extracts the singular parts of the integrand and treat them analytically [19], sigmoidal transformation which transforms the integrand to a periodic function [20] [21], and Duffy's transformation which cancels the singularity of type $\frac{1}{t}$ [22]. Most of these methods can be characterized in three categories: singularity subtraction, analytical transformation, and special purpose quadrature.

In this paper, we present an alternative approach for evaluating one dimensional singular integrals (weakly, strongly, and hyper-singular) which converge in the sense of Cauchy principal value and Hadamard finite part integrals. In addition, a proof of convergence of this method is outlined in Section 2 to serve as a theoretical basis for the method. In Section 3, the detailed implementations of our method are described for integrals over the standard interval $[-1,1]$ and arbitrary interval $[\mathrm{a}, \mathrm{b}]$.

\section{Approximation of Singular Integrals}

Let $S=\int_{D} f(x) \mathrm{d} x$ be a singular integral. Without loss of generality, the singularity can be assumed to be at zero. We assume that the integral $S$ has a finite value in the sense of principal value or Hadamard finite part integral, or in other words, this integral converges.

Let $D_{\epsilon}:=D \backslash B_{\epsilon}(0)$, where $B_{\epsilon}(0):=\{x:|x|<\epsilon\}$, where $\epsilon>0$. Then there exists numbers $\eta(n, \epsilon)$ and $a_{j}^{(\epsilon)}, 0 \leq j \leq n$, such that

$$
\left|\int_{D_{\epsilon}} f(x) \mathrm{d} x-\sum_{j=0}^{n} a_{j}^{(\epsilon)} \int_{D_{\epsilon}} U_{j}(x) \mathrm{d} x\right| \leq \eta(n, \epsilon) \rightarrow 0, \quad \text { as } n \rightarrow \infty,
$$

where $U_{j}, 0 \leq j \leq n$, are Chebyshev polynomials of second kind

$$
U_{j}(x)=\frac{\sin \left((j+1) \cos ^{-1}(x)\right)}{\sin \left(\cos ^{-1}(x)\right)}, \quad 0 \leq j \leq n .
$$

Since the integral $S$ converges, $\lim _{\epsilon \downarrow 0}\left|\int_{D_{\epsilon}} f(x) \mathrm{d} x-S\right|=0$. Fix $\epsilon_{0}>0$, there exists a $\delta\left(\epsilon_{0}\right)$ such that 


$$
\left|\int_{D_{\epsilon_{0}}} f(x) \mathrm{d} x-S\right|<\delta\left(\epsilon_{0}\right) \quad \forall \epsilon \leq \epsilon_{0} .
$$

For a fixed $\epsilon=\epsilon_{0}$, there exists an $n$ such that

$$
\left|\int_{D_{\epsilon_{0}}} f(x) \mathrm{d} x-\sum_{j=0}^{n} a_{j}^{\left(\epsilon_{0}\right)} \int_{D_{\epsilon_{0}}} U_{j}(x) \mathrm{d} x\right|<\delta\left(\epsilon_{0}\right) .
$$

Thus,

$$
\left|S-\sum_{j=0}^{n} a_{j}^{\left(\epsilon_{0}\right)} \int_{D_{\epsilon_{0}}} U_{j}(x) \mathrm{d} x\right|<2 \delta\left(\epsilon_{0}\right) .
$$

That means $S$ can be approximated by $\sum_{j=0}^{n} a_{j}^{\left(\epsilon_{0}\right)} \int_{D_{\epsilon_{0}}} U_{j}(x) \mathrm{d} x$.

Let us summarize the result.

Theorem 1. Let $S=\int_{D} f(x) \mathrm{d} x$ be a singular integral that converges. Suppose $x_{0}$ is its singularity in $D$. Then, for any $\epsilon>0$, there exists $\delta(\epsilon)>0, n$, and $a_{j}^{(\epsilon)}, 0 \leq j \leq n$, such that

$$
\left|S-\sum_{j=0}^{n} a_{j}^{(\epsilon)} \int_{D_{\epsilon}} U_{j}(x) \mathrm{d} x\right|<\delta(\epsilon), \quad D_{\epsilon}:=D \backslash B_{\epsilon}\left(x_{0}\right) .
$$

\section{Methods for Computing Singular Integrals}

\subsection{Integrals over $[-1,1]$}

In this section, we present a method for evaluating singular integral of the type

$$
S=\int_{-1}^{1} f(x) \mathrm{d} x
$$

For a more general integral, we will extend this method in Section 3.2.

From Section 2, we need to find the coefficient $a_{j}$ such that

$$
S \simeq \sum_{j=0}^{n-1} a_{j} \int_{-1}^{1} U_{j}(x) \mathrm{d} x,
$$

or

$$
f(x) \simeq \sum_{j=0}^{n-1} a_{j} U_{j}(x)
$$

Since $U_{j}$ are Chebyshev polynomials of second kind, they admit some nice properties

$$
\begin{gathered}
\text { 1. } \int_{-1}^{1} U_{j}(x) \mathrm{d} x=\frac{2 \sin ^{2}\left(\frac{j \pi}{2}\right)}{j+1}, \\
\text { 2. } \sum_{k=1}^{n} U_{j}\left(x_{k}\right) U_{i}\left(x_{k}\right)\left(1-x_{k}^{2}\right)=\frac{n}{2} \delta_{i j}, \quad \forall i, j<n,
\end{gathered}
$$

where $x_{k}, 1 \leq k \leq n$, are the roots of $U_{n}(x)$ in $[-1,1]$

$$
x_{k}=\cos \left(\frac{k \pi}{n+1}\right), \quad 1 \leq k \leq n \text {. }
$$


Now consider the following summation with $0 \leq i \leq n-1$

$$
\begin{gathered}
\sum_{k=1}^{n} f\left(x_{k}\right) U_{i}\left(x_{k}\right)\left(1-x_{k}^{2}\right)=\sum_{k=1}^{n} \sum_{j=0}^{n-1} a_{j} U_{j}\left(x_{k}\right) U_{i}\left(x_{k}\right)\left(1-x_{k}^{2}\right) \\
=\sum_{j=0}^{n-1} a_{j} \sum_{k=1}^{n} U_{j}\left(x_{k}\right) U_{i}\left(x_{k}\right)\left(1-x_{k}^{2}\right) \\
=\sum_{j=0}^{n-1} a_{j} \frac{n}{2} \delta_{i j} \\
=\frac{n}{2} a_{i}
\end{gathered}
$$

Thus, the coefficient $a_{i}$ can be computed by

$$
a_{i}=\frac{2}{n} \sum_{k=1}^{n} f\left(x_{k}\right) U_{i}\left(x_{k}\right)\left(1-x_{k}^{2}\right), \quad 0 \leq i \leq n-1,
$$

and

$$
S \simeq \sum_{i=0}^{n-1} a_{i} \int_{-1}^{1} U_{i}(x) \mathrm{d} x=\sum_{i=0}^{n-1} a_{i} \frac{2 \sin ^{2}\left(\frac{i \pi}{2}\right)}{i+1} .
$$

\subsection{Integrals over $[a, b]$}

In this section, we extend the method presented in Section 3.1 to singular integrals over arbitrary interval $[a, b]$

$$
S=\int_{a}^{b} f(x) \mathrm{d} x
$$

Note that

$$
\int_{a}^{b} U_{i}(x) \mathrm{d} x=\frac{b-a}{2} \int_{-1}^{1} U_{i}(y) \mathrm{d} y .
$$

Therefore, we just need to recompute the coefficients $a_{i}$ in this formula

$$
S \simeq \sum_{i=0}^{n-1} a_{i} \int_{a}^{b} U_{i}(x) \mathrm{d} x=\frac{b-a}{2} \sum_{i=0}^{n-1} a_{i} \int_{-1}^{1} U_{i}(y) \mathrm{d} y .
$$

Let $x \in[a, b]$ and $y \in[-1,1]$. Define

$$
y_{k}:=\cos \left(\frac{k \pi}{n+1}\right), \quad 1 \leq k \leq n,
$$

the roots of $U_{n}(y)$ in $[-1,1]$. Then, using this change of variable formula to find the corresponding collocation points $x_{k}$ in $[a, b]$

$$
x_{k}=\frac{b-a}{2} y_{k}+\frac{b+a}{2}, \quad 1 \leq k \leq n .
$$

Let

$$
f\left(x_{k}\right) \simeq \sum_{j=0}^{n-1} a_{j} U_{j}\left(y_{k}\right), \quad 1 \leq k \leq n .
$$

Then the coefficients $a_{i}$ can be computed as follows. Consider the following summation with $0 \leq i \leq n-1$ 


$$
\begin{gathered}
\sum_{k=1}^{n} f\left(x_{k}\right) U_{i}\left(y_{k}\right)\left(1-y_{k}^{2}\right)=\sum_{k=1}^{n} \sum_{j=0}^{n-1} a_{j} U_{j}\left(y_{k}\right) U_{i}\left(y_{k}\right)\left(1-y_{k}^{2}\right) \\
=\sum_{j=0}^{n-1} a_{j} \sum_{k=1}^{n} U_{j}\left(y_{k}\right) U_{i}\left(y_{k}\right)\left(1-y_{k}^{2}\right) \\
=\sum_{j=0}^{n-1} a_{j} \frac{n}{2} \delta_{i j} \\
=\frac{n}{2} a_{i}
\end{gathered}
$$

Thus

$$
a_{i}=\frac{2}{n} \sum_{k=1}^{n} f\left(x_{k}\right) U_{i}\left(y_{k}\right)\left(1-y_{k}^{2}\right), \quad 0 \leq i \leq n-1 .
$$

Finally,

$$
\begin{gathered}
S=\int_{a}^{b} f(x) \mathrm{d} x \\
\simeq \sum_{i=0}^{n-1} a_{i} \int_{a}^{b} U_{i}(x) \mathrm{d} x \\
=\frac{b-a}{2} \sum_{i=0}^{n-1} a_{i} \int_{-1}^{1} U_{i}(y) \mathrm{d} y \\
=\frac{b-a}{2} \sum_{i=0}^{n-1} a_{i} \frac{2 \sin ^{2}\left(\frac{i \pi}{2}\right)}{i+1} .
\end{gathered}
$$

\section{Conclusion}

In this paper, we investigated one dimensional singular integrals. A method is developed for evaluating weakly, strongly, and hyper singular integrals which converge in the sense of Cauchy principal value and Hadamard finite part integrals. The convergence of this method is proved and the detailed implementations are provided. One of the advantages of this method is that it is simple and relatively easy to implement. This method can serve as an alternative approach to other special methods in the literature.

\section{References}

[1] Ramm, A.G. (2013) Scattering of Acoustic and Electromagnetic Waves by Small Bodies of Arbitrary Shapes. Applications to Creating New Engineered Materials, Momentum Press, New York.

[2] Tran, N.T. (2017) Numerical Method for Solving Electromagnetic Scattering Problem by Many Small Impedance Bodies. Kansas State University, Manhattan.

[3] Ramm, A.G. and Tran, N.T. (2015) A Fast Algorithm for Solving Scalar Wave Scattering Problem by Billions of Particles. Journal of Algorithms and Optimization, 3, 1-13.

[4] Tran, N.T. (2013) Numerical Solution of Many-Body Wave Scattering Problem and Creating Materials with A Desired Refraction Coefficient. The International Journal of Structural Changes in Solids, 5, 27-38.

[5] Tran, N.T. (2017) Numerical Method for Solving Electromagnetic Wave Scattering 
by One and Many Small Perfectly Conducting Bodies. Kansas State University, Manhattan.

[6] Tran, N.T. (2016) Numerical Methods for Solving Wave Scattering Problems. Kansas State University, Manhattan.

[7] Chien, C.C., Rajiyah, H. and Atluri, S.N. (1991) On the Evaluation of Hyper-Singular Integrals Arising in the Boundary Element Method for Linear Elasticity. Computational Mechanics, 8, 57-70. https://doi.org/10.1007/BF00370548

[8] Karami, G. and Derakhshan, D. (1999) An Efficient Method to Evaluate Hypersingular and Supersingular Integrals in Boundary Integral Equations Analysis. Engineering Analysis with Boundary Elements, 23, 317-326.

https://doi.org/10.1016/S0955-7997(98)00085-X

[9] Neri, U. (1971) Singular Integrals and Sobolev Spaces. In: Singular Integrals, Springer, Berlin, 151-211. https://doi.org/10.1007/BFb0079059

[10] Wazwaz, A.M. (1997) A First Course in Integral Equations. World Scientific, Singapore.

[11] Hadamard, J. (2014) Lectures on Cauchy's Problem in Linear Partial Differential Equations. Courier Corporation, Westford.

[12] Resende, U.C., Moreira, F.J. and Pereira-Filho, O.M. (2007) Efficient Evaluation of Singular Integral Equations in Moment Method Analysis of Bodies of Revolution. Journal of Microwaves, Optoelectronics and Electromagnetic Applications (JMOe), 6, 373-391.

[13] Tzoulis, A. and Eibert, T.F. (2005) Review of Singular Potential Integrals for Method of Moments Solutions of Surface Integral Equations. Advances in Radio Science, 2, 93-99. https://doi.org/10.5194/ars-2-93-2004

[14] Hui, C.Y. and Shia, D. (1999) Evaluations of Hypersingular Integrals Using Gaussian Quadrature. International Journal for Numerical Methods in Engineering, 44, 205-214.

https://doi.org/10.1002/(SICI)1097-0207(19990120)44:2<205::AID-NME499>3.0.C $\underline{\mathrm{O} ; 2-8}$

[15] Tsamasphyros, G. and Dimou, G. (1990) Gauss Quadrature Rules for Finite Part Integrals. International Journal for Numerical Methods in Engineering, 30, 13-26. https://doi.org/10.1002/nme.1620300103

[16] Du, Q.K. (2001) Evaluations of Certain Hypersingular Integrals on Interval. International Journal for Numerical Methods in Engineering, 51, 1195-1210. https://doi.org/10.1002/nme.218

[17] Li, B. and Sun, W. (2009) Newton-Cotes Rules for Hadamard Finite-Part Integrals on an Interval. IMA Journal of Numerical Analysis, 30, 1235-1255. https://doi.org/10.1093/imanum/drp011

[18] Sun, W. and Wu, J. (2005) Newton-Cotes Formulae for the Numerical Evaluation of Certain Hypersingular Integrals. Computing, 75, 297-309. https://doi.org/10.1007/s00607-005-0131-5

[19] Guiggiani, M., Krishnasamy, G., Rudolphi, T.J. and Rizzo, F.J. (1992) A General Algorithm for the Numerical Solution of Hypersingular Boundary Integral Equations. Journal of Applied Mechanics, 59, 604-614. https://doi.org/10.1115/1.2893766

[20] Choi, U.J., Kim, S.W. and Yun, B.I. (2004) Improvement of the Asymptotic Behaviour of the Euler-Maclaurin Formula for Cauchy Principal Value and Hadamard Finite-Part Integrals. International Journal for Numerical Methods in Engineering, 61, 496-513. https://doi.org/10.1002/nme.1077

[21] Elliott, D. and Venturino, E. (1997) Sigmoidal Transformations and the Eu- 
ler-Maclaurin Expansion for Evaluating Certain Hadamard Finite-Part Integrals. Numerische Mathematik, 77, 453-465. https://doi.org/10.1007/s002110050295

[22] Duffy, M.G. (1982) Quadrature over a Pyramid or Cube of Integrands with a Singularity at a Vertex. SIAM Journal on Numerical Analysis, 19, 1260-1262. https://doi.org/10.1137/0719090 